\title{
El uso de discurso argumentativo en la enseñanza de ciencias: Un estudio exploratorio*
}

\author{
The use of argumentation in science teaching: An exploratory study \\ O uso do discurso argumentativo no ensino de ciências: Um estudo exploratório
}

\author{
Antonia Larraín ${ }^{\mathrm{a}}$, Paulina Freire ${ }^{\mathrm{b}}$

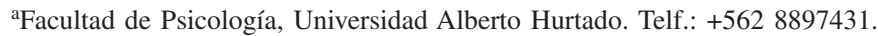 \\ Correo electrónico: alarrain@uahurtado.cl

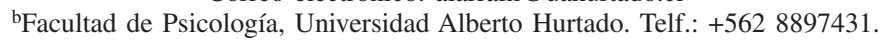 \\ Correo electrónico: pfreire@uahurtado.cl
}

\begin{abstract}
RESUMEN
Desde los años noventa existe un consenso creciente acerca de la importancia de la argumentación para el aprendizaje de las ciencias. Sin embargo, aunque existen varios estudios sobre argumentación en la enseñanza de ciencias, la mayoría de los estudios son de pequeña escala. El estudio que se reporta en este artículo pretendió aportar en la identificación de dimensiones para aprehender la argumentación en la enseñanza de ciencias en futuros estudios a gran escala. El estudio fue de tipo exploratorio en base al análisis de videos. Se analizaron ocho clases (cuatro de quinto básico y cuatro de séptimo). Participaron dos establecimientos subvencionados y tres profesores (un varón). Se realizaron análisis descriptivos cualitativos y cuantitativos. Los resultados muestran que para aprehender la argumentación en el aula de ciencias es necesario tener en cuenta tres dimensiones: nivel conceptual, estructura argumentativa y tipo de interacción.
\end{abstract}

Palabras clave: argumentación, discurso argumentativo, enseñanza de ciencias, discurso en sala de clase.

\begin{abstract}
Since the 1990s, there has been consensus about the relevance of argumentation for science teaching. However, although many studies have sought to study argumentation in the science classroom, most of them have been small in scale. This article presents an exploratory video study that aims to identify key dimensions to understand argumentation in science teaching, which can be useful in future large-scale studies. Three teachers (one male) from two State-subsidized schools participated. Eight lessons were video-recorded and analysed (grades five and seven). Results show that in order to grasp argumentation in science teaching it is important to take three dimensions into account: conceptual level, argumentative structure, and type of interaction.
\end{abstract}

Key words: argumentation, argumentative discourse, teaching science, classroom discourse.

\section{RESUMO}

Desde os anos noventa há um consenso crescente acerca da importância da argumentação para a aprendizagem de ciências. Contudo, ainda que existam vários estudos sobre argumentação no ensino de ciências, a maioria dos estudos é de pequena escala. O estudo que se apresenta neste artigo pretendeu contribuir com a identificação de dimensões para compreender a argumentação no ensino de ciências em futuros estudos de grande escala. O estudo foi do tipo exploratório em base à análise de vídeos. Analisaram-se oito aulas (quatro de quinta série e quatro de sétima série). Participaram dois estabelecimentos subvencionados e três professores (um homem). Realizaram-se análises

* Esta investigación contó con el financiamiento de Fondos Internos Universidad Alberto Hurtado. La escritura de este artículo fue posible gracias a Becas Chile Postdoctorado (CONICYT), y beca Postdoctorado Universidad Alberto Hurtado. 
descritivas qualitativas e quantitativas. Os resultados mostram que para apreender a argumentação na aula de ciências é necessário considerar três dimensões: nível conceitual, estrutura argumentativa e tipo de interação.

Palavras chave: argumentação, discurso argumentativo, ensino de ciências, discurso em sala de aula.

\section{INTRODUCCIÓN}

Desde fines de los años noventa existe un importante consenso entre los investigadores en enseñanza de ciencias respecto a que la argumentación es un tipo discursivo fundamental para el quehacer científico y que, por lo tanto, debe estar al centro de la enseñanza de ciencias (Candela, 1999; Driver, Newton y Osborne, 2000; Duschl y Osborne, 2002; Jimenez-Alexaindre y Erduran, 2008; Osborne, Erduran y Simon, 2004; Yore, Bisanz y Hand, 2003). Sin embargo, la evidencia internacional sugiere que la argumentación es un tipo discursivo poco presente en la enseñanza (Alexander, 2001) y, particularmente, en la enseñanza de ciencias (Roth, Druker, Garnier, Lemmens, Chen, Kawanaka, et al. 2006). Aunque no habría razones para pensar que existe una tendencia radicalmente diferente en las aulas chilenas, es importante constatarlo. Sobre todo considerando que los resultados de las pruebas internacionales en las que Chile ha participado que miden alfabetización científica (PISA 2000, 2006 y 2009) y manejo conceptual en ciencias (TIMSS 1999 y 2003), muestran que los estudiantes tienen desempeños modestos en comparación con los países desarrollados (Larraín, 2009; Mineduc, 2010).

Por otro lado, aunque existe un monto extensivo de investigación en argumentación en la enseñanza de ciencias, estos estudios se han centrado en la calidad de las habilidades argumentativas de los estudiantes (i.e. Kelly y Chen, 1999). Adicionalmente la mayoría de estos estudios son de pequeña escala y requieren de procesos largos y complejos de entrenamiento para la codificación y análisis, lo que hace poco factible la posibilidad de generalización de los resultados. Entonces, si tal y como plantea Erduran (2008), la necesidad de informar la práctica pedagógica depende de estudios a larga escala, es necesario realizar este tipo de estudios. Sin embargo, éstos requieren de desarrollos metodológicos apropiados que permitan analizar corpus amplios sin perder la posibilidad de aprehender los procesos discursivos en toda su complejidad.

El estudio que se presenta en este artículo pretende contribuir en esta tarea. Su objetivo fue identificar dimensiones relevantes para estudiar el uso del discurso argumentativo en la enseñanza de ciencias a gran escala. Para esto se analizaron ocho clases de ciencias en dos establecimientos educacionales subvencionados, y dos niveles de enseñanza: quinto y séptimo básico. A continuación se presentan y discuten antecedentes acerca de la relación entre argumentación, aprendizaje de ciencias y discurso en aula. Luego se presenta la metodología del estudio y los principales resultados. Se finaliza recogiendo aquellas dimensiones que aparecieron como relevantes en el uso de discurso argumentativo en las aulas observadas. 


\section{ANTECEDENTES TEÓRICOS}

\subsection{ARGUMENTACIÓN Y DISCURSO ARGUMENTATIVO}

Argumentación, tal y como lo plantea Jiménez-Aleixandre y Erduran (2008), es un término polisémico que tiene diferentes significados según la aproximación teórica desde la que se la conciba. Aunque no es posible discutir (como sería apropiado) las diferentes nociones y énfasis en relación a la concepción de argumentación, es necesario hacer una discusión mínima que permita plantear qué se entenderá por argumentación y discurso argumentativo en este artículo.

Van Eemeren y Grootendorst (1992) sostienen una noción de argumentación en la cual la controversia y la discusión crítica de puntos de vista contrarios son centrales. La argumentación aparece toda vez que dos o más personas sostienen puntos de vista opuestos e intentan llegar a un acuerdo. Ahora bien, aunque estos autores enfatizan el aspecto controversial de la argumentación no es claro en qué sentido esta actividad podría ocurrir cuando es una misma persona quien sostiene y discute una idea. Al tener en mente una discusión racional y crítica ideal, los autores se centran en la argumentación interpersonal sin dar cuenta de procesos de argumentación donde es una misma persona quien propone y opone. Desde un punto de vista psicológico, estos últimos procesos de argumentación son de gran interés.

Consecuentemente, incluso enfatizando el aspecto controversial en la definición de argumentación, y bajo una noción dialógica del discurso (Bajtín, 2002) y de la argumentación (Billig, 1987; Leitão, 2000), adherimos a la idea de que esta aparece en cada actividad discursiva donde se formulan dos puntos de vista opuestos que intentan llegar a un entendimiento, independiente de si es la misma persona quien propone y opone.

Más aún, y siguiendo la aproximación de Leitão (2000), diremos que la argumentación no está presente solamente cuando las posiciones opuestas que se intentan resolver están explícitamente formuladas, sino que basta una justificación para que una posición alternativa aparezca de manera implícita. La necesidad de justificación aparece sólo cuando hay una noción de la debilidad de una opinión, la que siempre es relativa a la fortaleza de opiniones alternativas. De esta manera, sólo la aparición de la justificación transformaría un discurso en argumentativo (Toulmin, 1958; Perelman y Olbrechts-Tyteca, 1969).

Dicho esto, evidentemente no es lo mismo una interacción sólo con justificación, que una interacción con justificación y posiciones opuestas formuladas explícitamente. De hecho, para Leitão (2000) la unidad argumentativa es el conjunto de una posición justificada, otra posición opuesta justificada (contra-argumento) y una tercera posición o respuesta. En este sentido, si bien sólo la justificación convierte a un discurso en argumentativo, para la autora es posible describir distintos mecanismos semióticos que conformarían la unidad del proceso argumentativo: argumento, contra-argumento y respuesta.

En un primer momento, cuando no hay ningún mecanismo semiótico de los descritos por Leitão (2000), el discurso no es argumentativo. Cuando hay una justificación o una demanda por ésta, se provoca la aparición de al menos dos posiciones: la que se sostiene y debe ser fundamentada, y aquella que virtualmente podría competir con la primera. Es decir, la justificación abre el discurso a la presencia de posiciones alternativas, aunque éstas no estén explícitamente formuladas. Luego, en un segundo momento y con la presencia del contra-argumento, aparece una oposición explícita dirigida al argumento (posición 
más justificación) anteriormente esgrimido. Este movimiento según Leitão (2000) fuerza a la consideración y revisión de la posición sostenida y sus fundamentos. Por último, la presencia de un tercer movimiento, la respuesta, es el mecanismo semiótico que permite la evaluación de ambas posiciones y la formulación de un tercer movimiento.

De esta manera y para efectos de este estudio, consideramos el discurso argumentativo como todo discurso en donde al menos aparece una justificación o demanda por ella, pudiendo ésta verificarse en una dimensión interpersonal o en el discurso de un mismo hablante. Coherentemente, el estudio se propuso describir todo momento en el que apareciera cualquier indicación de discurso argumentativo en el aula de ciencias de manera de comprender su complejidad y aprehender dimensiones relevantes para su estudio a larga escala.

\subsection{ARGUMENTACIÓN, APRENDIZAJE DE CIENCIAS Y DESARROLLO DE HABILIDADES ARGUMENTATIVAS}

La capacidad de apoyar y contrarrestar ideas es fundamental para el aprendizaje escolar pues promueve, por una parte, la negociación y co-construcción de conocimiento y, por otra, procesos meta-cognitivos (Leitão, 2002). De hecho, Schwartz (2009), recogiendo la literatura psicológica en el tema, plantea tres razones por las que la argumentación contribuye al aprendizaje: 1. El proceso de expresar verbalmente las propias ideas, anticipando posibles puntos de vista alternativos, tendría un efecto en la examinación de las propias teorías que promueve la comprensión y construcción de conocimiento; 2 . La multiplicidad y oposición que involucra el defender y contraponer puntos de vista invita a explorar la validez de las ideas y evaluarlas de manera sistemática; 3. Promueve la organización del conocimiento de tal manera que disminuye el nivel de carga cognitiva: la tarea de proponer y contraponer es compartida por distintos individuos.

Ahora bien, la relación entre argumentación y aprendizaje de ciencias no es una hipótesis meramente teórica. Existen diversos estudios tanto con niños en edad escolar como universitarios, que muestran el efecto de la argumentación en el aprendizaje. Un grupo de estudios reportados en Howe (2010) ha mostrado la relación entre la discusión en grupos en la enseñanza de ciencias entre niños de 8-12 años y el desarrollo conceptual en ciencias. El paradigma de estos estudios corresponde a diseños experimentales prepost en los que los estudiantes trabajan en grupos pequeños para resolver problemas que involucran conceptos elementales como: densidad y flotación, velocidad o temperatura. Las instrucciones fuerzan a los grupos a levantar y discutir hipótesis, testearlas y llegar a un acuerdo acerca de su explicación. Todos estos estudios confirman el efecto de la discusión en el desarrollo conceptual de los estudiantes. Más aún, Howe et al. (2007) mostraron que el predictor más importante de la ganancia conceptual de los estudiantes fue la expresión de opiniones contrarias. Este efecto fue detectado aún en post-test aplicados 18 meses después. Otro grupo de estudios realizados en el contexto del programa Thinking Together liderado por Neil Mercer en el Reino Unido, con niños edad similar a los estudios de Howe (2010), también muestra ganancias en el aprendizaje, uso de discurso argumentativo en el aula y desarrollo de pensamiento general, cuando se argumenta en el aula (Mercer et al., 2004; Mercer 2009). Estudios con estudiantes universitarios son coherentes con los hallazgos anteriormente descritos (Asterhan y Schwarz, 2007; 2009; 
Nussbaum y Sinatra, 2003), mostrando que cuando los estudiantes argumentan y discuten sus ideas tienen significativamente más ganancias conceptuales.

Por otro lado, estudios en el desarrollo de argumentación a partir de intervenciones en que se enseña a argumentar explícitamente (Zohar y Nemet, 2002), o en el contexto de actividades instruccionales (Kuhn y Udell, 2003; Osborne et al., 2004), muestran una importante tendencia a un aumento de la cantidad y cualidad de discurso argumentativo de los estudiantes. De hecho, diversos estudios muestran cómo la educación formal en el segundo ciclo básico (niños entre diez y catorce años), sí puede hacer una diferencia en el desarrollo de habilidades argumentativas de los estudiantes (De Chiaro y Leitão, 2005; Hammer et al., 2008; Kuhn y Udell, 2003; Rojas-Dummond y Peon, 2004). Siguiendo esta idea, es relevante preguntarse si la escolaridad promueve hoy en día el desarrollo de la argumentación: ¿Se usa la argumentación en la enseñanza en general y, en particular, en ciencias?

\subsection{DISCURSO EN SALA DE CLASES Y ARGUMENTACIÓN}

La investigación en discurso en sala de clases a gran escala en general (no particularmente en ciencias), tanto en contextos anglosajones (Cazden, 1991; Galton et al., 1999; Nystrand et al., 1997) como de investigación comparada (Alexander, 2001; Stigler et al., 1999), muestra desde hace un tiempo que el discurso docente que predomina en las aulas no es propicio para el aprendizaje y desarrollo cognitivo. Dos estudios a partir de la evidencia del Programa Nacional de Evaluación Docente en el área de lenguaje y matemáticas (Preiss, 2009; Preiss, 2010) sugieren que el discurso que predomina en sala de clases chilenas no es la excepción.

Particularmente en ciencias, la impresión extendida entre investigadores educacionales francófonos (Burton y Flammang, 2001) y anglosajones (Duschl y Osborne, 2002) es que predomina una teoría empiricista más centrada en la experimentación que en la discusión. Coherente con esta visión, el estudio de video del TIMSS 1999 en ciencias concluye que: "Las discusiones donde los estudiantes jugaban un rol más central, como aquellas descritas extendidamente como "formas discursivas altamente interactivas" (Schoenfeld, 2002), "discurso argumentativo" (Nelly y Chen, 1999), "enseñanza diagnóstica” (Bell y Purdy, 1985) y "conversaciones científicas" (Gallas, 1995), fueron raramente observadas" (Roth et al., 2006:111).

Aunque, como se mencionó anteriormente, no habrían razones para pensar que en nuestro país la situación es diferente, no existen estudios a larga escala que den cuenta del tipo de discurso predominante en la enseñanza de ciencias. Menos aún existe evidencia acerca del uso de discurso argumentativo presente en la educación de nuestro país. Esto último no sólo responde a una carencia de investigación nacional en el área, sino más bien a una tendencia mundial a estudiar la argumentación en la enseñanza de ciencias a pequeña escala. Por otro lado, aunque existe considerable investigación en argumentación en la enseñanza de ciencias, muchos de estos estudios se han centrado en las habilidades argumentativas de los estudiantes (Jiménez-Aleixandre, Rodrigues y Duschl, 2000; Kelly, Druker y Chen, 1998; Kim y Song, 2006) con un especial foco en su calidad (Osborne et al., 2004; Sandoval y Millwood, 2005) y condiciones de desarrollo (Kuhn y Udell, 2003; Rojas-Dummond y Peon, 2004). El estudio de la argumentación tal y como ocurre espontáneamente en el aula ha sido abordado en menor medida. 
En síntesis, si bien el fomento de la argumentación para el aprendizaje de ciencias es relevante, hoy en día no se cuenta con datos acerca de cómo ésta ocurre espontáneamente en el aula. Se necesitan estudios a larga escala que provean descripciones cualitativas y cuantitativas que informen apropiadamente las políticas públicas. Sin embargo, para aprehender la riqueza y diversidad del discurso espontáneo en análisis a larga escala se requiere contar con indicadores que permitan describirlo de manera pertinente. Justamente éste es el objetivo del estudio que se describe a continuación: identificar dimensiones relevantes para estudiar el uso de discurso argumentativo en la enseñanza de ciencias a gran escala.

\section{MARCO METODOLÓGICO}

\subsection{DISEÑO Y PARTICIPANTES}

El estudio fue un estudio exploratorio-descriptivo de video a partir de evidencia audiovisual (videos de aula) recogida especialmente para este estudio. Participaron tres docentes que enseñan en dos establecimientos educacionales de la Región Metropolitana: Uno ubicado en el sector sur-oriente de Santiago y otro en el sector poniente de la capital. Ambos establecimientos son subvencionados, siendo el primero particular y el segundo municipal. Fueron escogidos teniendo en cuentas ciertos indicadores que hacían pensar que el uso de la argumentación fuera relativamente frecuente (tomando en cuenta que éste según la evidencia internacional es más bien escaso). Estos indicadores fueron, para el caso del establecimiento particular, tener un puntaje alto en el Simce de cuarto y octavo básico en ciencias en los últimos cinco años. En el caso del establecimiento municipal el criterio de selección fue la pertenencia al programa Ecbi del Mineduc, programa que promueve la enseñanza de ciencias a través de la indagación.

Participaron los profesores a cargo de la enseñanza de ciencias en quinto y séptimo básico de cada establecimiento. En el caso del colegio particular dos profesores distintos (una mujer) enseñaban en estos niveles. El docente, de 35 años, tenía formación basal en ciencias biológicas (mención en biología celular) y al momento del estudio estaba finalizando su formación en pedagogía en biología. La profesora, de 42 años, con formación inicial en pedagogía básica, había desarrollado posteriormente un curso universitario de perfeccionamiento en el área de las ciencias. Ambos profesores llevaban aproximadamente un año trabajando en ese colegio.

En el caso del establecimiento municipal una profesora enseñaba en ambos niveles. Al momento del estudio tenía 50 años y se desempeñaba hace 10 como profesora de ese colegio. Su formación inicial era en pedagogía básica y había tenido cursos de especialización y perfeccionamiento en Estudio y Comprensión de la Naturaleza, y metodologías de indagación.

Los profesores escogieron las clases que serían observadas y registradas en video (unidades temáticas). En total once clases fueron filmadas: tres del profesor del establecimiento particular, tres de cada nivel en el establecimiento municipal, y dos de la profesora del colegio particular. Se filmaron clases consecutivas pertenecientes a una misma unidad. Finalmente participaron en los análisis dos sesiones de cada nivel (en total 8). Fueron excluidas una del profesor del establecimiento particular y una de cada nivel 
del establecimiento municipal con el fin de analizar igual número de sesiones en cada nivel. Las exclusiones siguieron un criterio de calidad de registro de audio, es decir, se excluyeron las sesiones que tenían problemas o dificultades en este aspecto.

\subsection{PROCEDIMIENTOS}

Se contactó a los directores de los establecimientos para explicarles el estudio e invitarlos a participar. Ambos directores accedieron obteniendo su consentimiento. Luego de esto, se procedió a contactar a los profesores y explicarles del estudio y su participación. Se les ofreció una retroalimentación y los videos de sus clases. Una vez que los profesores estuvieron de acuerdo con participar se procedió a pedirles que escogieran fechas y, por tanto, unidades temáticas que les fueran cómodas para ser observadas y registradas. Se coordinaron las visitas y se realizaron las observaciones. Dos integrantes del equipo de investigación filmaron las clases los días acordados. Estas fueron digitalizadas con el objetivo de facilitar los análisis.

\subsection{ANÁLISIS}

La primera aproximación a los datos se hizo rastreando los marcadores que tradicionalmente se utilizan como indicadores de argumentación, entre los que se encuentran: las preguntas "por qué", las respuestas que contienen justificaciones (porque), preguntas por acuerdos o desacuerdos (“¿Están todos de acuerdo?”), los comentarios con respecto al grado de acuerdo o desacuerdo con la opinión de otro ("Yo no estoy de acuerdo"), entre otros. Se optó por partir el análisis rastreando este tipo de marcadores de manera de apreciar el discurso argumentativo presente en los datos y extraer de éstos las dimensiones relevantes para su caracterización y descripción.

Un segundo momento del análisis fue sistematizar las dimensiones que aparecieron relevantes para describir de manera cuantitativa el tipo de discurso argumentativo presente en el corpus. A continuación se presentan los resultados en dos partes. La primera describe la lógica de extracción de las dimensiones relevantes y la segunda, cuantitativamente, el discurso argumentativo presente en las clases observadas.

\section{RESULTADOS}

\subsection{DIMENSIONES PARA APREHENDER DISCURSO ARGUMENTATIVO}

El marcador argumentativo más común en las clases observadas fue la pregunta justificativa "por qué". Generalmente a cargo del docente, esta pregunta aparece mucho más que cualquier otro marcador iniciando procesos de discurso argumentativo. Sin embargo, fue evidente a poco andar que estas preguntas eran de muy distinta naturaleza, tanto a nivel conceptual, argumentativo y del tipo de interacción. Esto se puede apreciar en la Tabla 1 donde se presentan algunas interacciones que contienen este tipo de marcador. 


\begin{tabular}{|c|c|}
\hline 1 & $\begin{array}{c}\text { P: ¿Cuál te agrado más a ti? } \\
\text { E1: El azúcar } \\
\text { P: ¿Por qué? ¿Por qué te agradó más ese? } \\
\text { E1: No sé } \\
\text { P: Uno tiene que saber por qué le agradan las cosas... ya ¿a usted? } \\
\text { E2: El azúcar porque es dulcesito y sabe bien. } \\
\text { P: Ya, bien. ¿Cuál fue el sabor que más les agradó? } \\
\text { EE: Azúcar } \\
\text { P: Oye mira, ¿Todos coinciden? ¿Por acá? } \\
\text { E3: La sal } \\
\text { P: Mira, a ella le agrada la sal } \\
\text { EE: Buah }\end{array}$ \\
\hline 2 & $\begin{array}{c}\text { P: ¿Tú maduras físicamente y psicológicamente? } \\
\text { SS: Sí } \\
\text { P: ¿Por qué? A ver, ¿Por qué dicen que ustedes maduran psicológicamente y físicamente? } \\
\text { ¿Por qué dicen que maduran? A ver, Ricardo. } \\
\text { E1: Porque con más responsabilidades se tienen que ir tomando otros aspectos de las } \\
\text { situaciones, otra manera de pensar, otra manera de hacer las cosas. } \\
\text { P: Ya ¿y vas a ser un adulto verdad? ¿Y para llegar a adulto como tienes que llegar, Ricardo? } \\
\text { E1: Tiene que ser un pensamiento totalmente radical al de ser un niño. } \\
\text { Ya. ¿Tú podrías ser adulto teniendo un cuerpo de niño? } \\
\text { EE: No } \\
\text { P: Oye, pero mira, piensen la pregunta que estoy haciendo ¿Tú podrías llegar a ser adulto } \\
\text { teniendo un cuerpo de un niño de seis a nueve años? ¿Entonces tienes que cambiar para ser } \\
\text { adulto físicamente? Porque te crece el pene, te crecen los testículos, eres un hombrecito } \\
\text { cuando adulto con otra carita... no puedes tener una carita de un niño de seis a nueve años } \\
\text { ¿verdad? (se interrumpe la interacción) }\end{array}$ \\
\hline 3 & $\begin{array}{c}\text { P: Ya, y hay una glándula que es responsable de todos estos cambios que suceden en la } \\
\text { pubertad, ¿Cuál será? } \\
\text { E1: La hipófisis. } \\
\text { P: Bien Gerardo, la hipófisis, y ¿Por qué se relaciona con la aparición de estas } \\
\text { características? } \\
\text { EE.... }\end{array}$ \\
\hline 4 & $\begin{array}{l}\text { :Y ¿Se acuerdan que hablamos de los autótrofos y heterótrofos? ¿Quiénes eran autótrofos? } \\
\text { E1: Las plantas } \\
\text { P: Ya, las plantas y ¿Por qué las plantas son autótrofas? } \\
\text { E1: Porque se alimentan por sí mismas (..) } \\
\text { P: ¿Porque se alimentan por si mismas? (...)Ya. }\end{array}$ \\
\hline 5 & $\begin{array}{l}\text { P: (...)Las deformaciones irreversibles también se llaman plásticas, por tanto ¿Cuál sería el } \\
\text { otro tipo de deformación? } \\
\text { E1: Reversible } \\
\text { P: La contraria, la reversible que también se podría llamar elástica. ¿Por qué? Porque actúa } \\
\text { igual que el elástico y aplico una fuerza al elástico y cambia su forma, se estira, pero cuando } \\
\text { quito esa acción de la fuerza el elástuico vuelve a su forma } \\
\text { E2: Pero el elástico se puede romper } \\
\text { P: Pero ese es otro asunto. Si le aplico más fuerza que la Resistencia propia del material } \\
\text { obviamente puede romperse, todo material que lo lleve a su extremo puedo obtener que se } \\
\text { rompa. }\end{array}$ \\
\hline
\end{tabular}




\begin{tabular}{|c|c|}
\hline 6 & $\begin{array}{c}\text { P: ¿Cuántos grupos de fichas se pueden formar? } \\
\text { E1: } 5 \\
\text { P: ¿Estamos todos de acuerdo que se formaron 5? } \\
\text { EE: Noo } \\
\text { E2: Cuatro } \\
\text { P: A ver, Valeria ¿Por qué dices tú cuatro? } \\
\text { E2: Porque hay olor, color, vista, comida. } \\
\text { P. ¿La comida es un sentido? } \\
\text { EE: Noo } \\
\text { P: ¿Cuál es el sentido? } \\
\text { E3: Gusto } \\
\text { P: Ya, entonces tenemos cuatro, ¿Por qué decimos cinco? } \\
\text { E1: porque faltó el tacto } \\
\text { P: Ah! El tacto, entonces ¿Cuántos son, Valeria? } \\
\text { E2: Cinco } \\
\text { P: ¿Están todos de acuerdo que se formaron cinco grupos? } \\
\text { EE: Sii }\end{array}$ \\
\hline 7 & $\begin{array}{c}\text { P: Ya. Aquí tenemos sus opiniones del inicio de la clase. Dice, la pubertad es la etapa de } \\
\text { desarrollo que tiene más cambios. Están de acuerdo con esto? } \\
\text { EE: Sii } \\
\text { E1: No } \\
\text { P: A ver, Pablo, ¿Por qué crees que no es la etapa de mayor cambio? Cuál sería entonces? } \\
\text { E1: Porque en la gestación es la etapa que hay más cambios en poco tiempo } \\
\text { P: ¿El resto está de acuerdo? Porque antes pensaban que la pubertad era la de mayor cambio. } \\
\text { Ah! Ahora todos están de acuerdo? } \\
\text { EE: Sii } \\
\text { P: Bien, ya (...) }\end{array}$ \\
\hline 8 & $\begin{array}{c}\text { P: ¿Cuál es el primer cambio en la pubertad? } \\
\text { E1: El vello púbico } \\
\text { P: ¿Ese es el primer cambio dices tu? } \\
\text { E2: Nooo }\end{array}$ \\
\hline
\end{tabular}

Tabla 1. Interacciones discursivas que contienen preguntas Por qué

\subsubsection{Dimensión conceptual}

Lo primero que aparece es que las interacciones descritas en la Tabla 1, si bien ocurren todas en el contexto de enseñanza de ciencias, involucran un trabajo conceptual cualitativamente distinto.

Encontramos diferencias según el uso o no de conceptos propios de la ciencia. Mientras la interacción 1 de la Tabla 1 no involucra conceptos científicos y trabaja con concepciones cotidianas (i.e. sabores), las interacciones restantes sí involucran conceptos propios de las ciencias (deformaciones reversibles, estímulo, pubertad). Ahora bien, incluso en las interacciones que involucran conceptos científicos seguimos encontrando diferencias en el trabajo conceptual realizado. Por ejemplo, en la interacción 2 de la Tabla 1, la demanda de justificación de la profesora provoca un trabajo en el que los conceptos científicos no se relacionan sistemáticamente entre sí al modo de la definición 
o taxonomía. En este caso, la maduración psicológica y física se liga a la experiencia personal ("Porque con más responsabilidades se tienen que ir tomando..."), lo que resulta en una descripción de lo que sería en términos de sentido común la maduración psicológica y física. Lo interesante es que en esta interacción la misma pregunta pudo ser trabajada ligando la maduración psicológica y física con otros conceptos científicos de manera sistemática. Por el contrario, en la interacción 3 de la Tabla 1, se observa un trabajo en el que los conceptos científicos se relacionan sistemáticamente (hipófisis y características secundarias).

De esta manera, observamos dos formas de trabajar con conceptos propios de la ciencia. La primera se caracteriza por trabajar conceptos científicos sin ligarlos sistemáticamente entre sí (por ejemplo, al modo de la definición). La segunda se caracteriza por trabajar los conceptos científicos relacionados entre sí y definidos según propiedades generales.

Estas características, el involucramiento de conceptos científicos y el uso taxonómico de éstos, dan paso a la distinción de tres niveles en el trabajo conceptual:

1) 'No conceptual': se caracteriza por un uso del lenguaje cotidiano con ausencia de conceptos científicos.

2) 'Conceptual no taxonómico': se caracteriza porque los conceptos científicos no se usan relacionados entre sí desde sus propiedades generales y mecanismos que subyacen la relación.

3) 'Taxonómico': se caracteriza por un uso sistemático de relaciones entre conceptos científicos según las propiedades de la clase a la que pertenecen.

\subsubsection{Dimensión argumentativa}

Los distintos usos del "por qué" también evidenciaron una diversidad que por momentos hacía difícil el reconocimiento de procesos de argumentación. Esto se clarificó una vez que se apreció la aparición de distintas estructuras argumentativas según la presencia o ausencia de los mecanismos semióticos propuestos por Leitão (2000). No todo el discurso argumentativo apareció como una unidad que involucraba justificación, oposición y respuesta. Por el contrario, en muchos momentos sólo apareció la demanda por justificación sin que fuese claro cuáles eran las posiciones alternativas o el tema que se quería resolver. Por ejemplo, en la Tabla 1 en la interacción 3 la profesora pregunta por qué la hipófisis se relaciona con la aparición de las características secundarias, sin obtener respuesta. La ausencia de respuesta no permite entablar un diálogo argumentativo en el que ambos están interesados en llegar a un acuerdo. Sin embargo, igualmente la pregunta abre una multiplicidad de posibles razones por las cuales la hipófisis es responsable de los cambios de la pubertad, haciendo evidente la necesidad de dar apoyo adicional a la idea en forma de explicación (relación causal) y no sólo de dar cuenta de la relación. En esta interacción, el estudiante no da cuenta de ninguna explicación pero la necesidad queda presente en la interacción.

Por otro lado, encontramos interacciones que contienen todos los movimientos implicados en la unidad de Leitão (justificación, oposición y respuesta), pero de manera particular. Por ejemplo, en la interacción 1 de la Tabla 1 la profesora pregunta a dos estudiantes primero por qué les agradó más el azúcar. Al hacerlo, demanda justificación de una preferencia, cuya alternativa está igualmente ligada a gusto personal más que a razones de otro tipo. Sin embargo, aunque aparece una justificación que es difícil de 
evaluar como fuerte o débil ("porque es dulcesito y sabe bien"), aparece una posición distinta que no se justifica ("la sal”). Además, inmediatamente el grupo reacciona oponiéndose a su vez a esta opinión sin tampoco justificar ("Buah"), por lo que no alcanza a ser más que una oposición (es decir, no alcanza a ser contra-argumento). Finalmente la profesora cierra la interacción con una respuesta. Aunque en esta interacción aparecen los movimientos descritos por Leitão, éstos se verifican de manera muy particular y alejada de la estructura argumentativa prototípica (opinión con justificación, contra-opinión justificada, respuesta). La estructura puede ser entendida como opinión sin justificación (s/j)+opinión con justificación+opinión alternativa s/j+oposición s/j+respuesta. Se aprecia cómo aparece discurso argumentativo a propósito de cuestiones de sentido común que no ofrecen una oportunidad evidente para argumentar.

En síntesis, se evidenció la existencia de una diversidad en los tipos de uso de discurso argumentativo, pudiendo distinguirse distintas estructuras caracterizadas por la presencia (o ausencia) y forma de los mecanismos semióticos propuestos por Leitão.

Ahora bien, habiendo diferenciado la dimensión conceptual y argumentativa, es interesante observar que la combinación de estas dimensiones dan cuenta de una diversidad aún mayor de tipo de interacciones argumentativas. Por ejemplo, observamos que la interacción 5 de la Tabla 1, coincide con una interacción de alto nivel conceptual (tal y como fue definida anteriormente), y con una estructura 'prototípica' de argumentación. En esta interacción tenemos al profesor quien en su tercera intervención provee de un sinónimo a la transformación reversible (elástica) luego de lo cual demanda una justificación que él mismo, a reglón seguido, desarrolla (“¿Por qué? Porque actúa igual que el elástico y aplico una fuerza al elástico y cambia su forma"). Luego un estudiante reacciona poniendo una situación que debilita la justificación entregada por el profesor. Esta reacción no es sólo una opinión sino actúa como contra-argumento. Finalmente, el profesor cierra la interacción de manera autoritativa poniendo en su lugar el contra-argumento como un caso que depende de otros factores y no afecta la justificación inicial.

A diferencia de la interacción anterior, la interacción 1 de la Tabla 1 es de bajo nivel conceptual y presenta una estructura argumentativa menos 'prototípica', como ya fue discutida. Esto podría sugerir que las estructuras argumentativas 'prototípica' siempre van de la mano con trabajo de alto nivel conceptual (ver interacción 6, Tabla 1). Claramente esto no tiene apoyo en las interacciones estudiadas. En las clases observadas también ocurrieron interacciones de alto nivel conceptual con estructuras argumentativas básicas (interacción 3, Tabla 1) y, mucho más frecuentemente, interacciones de bajo nivel conceptual en las que estaban en juego diferentes opiniones tal y como es posible apreciar en la interacción 6 de la Tabla 1. En esta interacción hay opinión inicial (5), oposición justificada ("Noo, 4 [...] Porque hay olor, color, vista, comida"), contra-argumento dirigido a esta oposición ("porque faltó el tacto") y respuesta (que es formulada por la misma estudiante que inicialmente insistió en que eran cuatro grupos y no cinco). Más aún, aunque esta interacción es de bajo nivel conceptual, se evidencia un cambio de opinión favorable para el aprendizaje en esta estudiante.

\subsubsection{Dimensión interaccional}

Por último, fue posible observar que los episodios argumentativos variaban según el tipo de participantes involucrados y las relaciones entre ellos. Por una parte, en la 
instrucción directa el mismo docente puede justificar su postura, o exponer distintos puntos de vista y resolverlos. Por otra parte, el docente puede sostener diferencias de opinión con un estudiante (interacción 5, Tabla 1) o el profesor puede representar una posición con respecto a la cual algunos estudiantes difieren y/o concuerdan (interacción 7, Tabla 1). En conclusión, hay interacciones en las que las estructuras argumentativas son sostenidas por un mismo hablante o por dos o más hablantes.

De acuerdo a lo observado, es posible describir también interacciones en las que los estudiantes no se oponen a lo planteado por el profesor (lo que sucede en la interacción 5, Tabla 1), sino a lo sostenido por otros estudiantes (interacciones 1, 6 y 7, Tabla 1). Se identificaron, entonces, cinco estructuras de interacción que se detallan en la Tabla 2.

\begin{tabular}{|c|c|}
\hline & Definición \\
\hline IRE & $\begin{array}{c}\text { Interacción iniciada por pregunta docente, contribución o respuesta de un } \\
\text { estudiante (o de toda la clase al unísono) y evaluación docente }\end{array}$ \\
\hline IRF & $\begin{array}{c}\text { Involucra pregunta docente, una o más contribuciones por parte de un } \\
\text { mismo estudiante (o de la clase completa sin distinción de contribuciones } \\
\text { individuales) y seguimiento de parte del profesor más que una evaluación }\end{array}$ \\
\hline IRD & $\begin{array}{c}\text { Interacción iniciada por pregunta docente, contribuciones de distintos } \\
\text { estudiantes dirigidos al planteamiento docente y seguimiento docente }\end{array}$ \\
\hline IRI & $\begin{array}{c}\text { Interacción iniciada por pregunta docente, contribuciones de distintos } \\
\text { estudiantes dirigidos a planteamientos de otros estudiantes (no sólo a la la } \\
\text { posición del profesor) y seguimiento docente. }\end{array}$ \\
\hline DIR & Episodios de instrucción directa donde el profesor expone contenido. \\
\hline
\end{tabular}

Tabla 2. Tipo de interacciones observadas

Cabe destacar en estas interacciones, que las diferencias en el tipo de seguimiento que puede hacer el docente frente a las respuestas de los estudiantes determinan también distintos tipos de procesos argumentativos. Por ejemplo, frente a la respuesta del alumno a una demanda de justificación, el docente puede hacer seguimiento a la pregunta solicitando mayor elaboración al mismo estudiante (interacción 2, Tabla 1) o a otros estudiantes (como ocurre en la interacción 6, Tabla 1). En este último caso, el docente usa la respuesta como oportunidad para que los estudiantes piensen. Por otro lado, el profesor puede formular un contra-argumento a la respuesta del estudiante para forzarlo a elaborar más su respuesta, o puede formularlo como una respuesta de autoridad que cierra la interacción (interacción 5, Tabla 1). Por último, frente a una diferencia de opinión entre los estudiantes, el docente en lugar de demandar justificación de ambas posiciones y abrir la discusión puede cerrar la interacción inhibiendo la aparición de discurso argumentativo (tal y como ocurre en la interacción 8, Tabla 1).

En su conjunto, entonces, los datos sugieren que para aprehender el tipo de discurso argumentativo que ocurre en la enseñanza de ciencias es necesario tener en cuenta el cruce de tres dimensiones: nivel de uso conceptual (cómo y para qué se usan los conceptos científicos), mecanismos semióticos (involucrados en unidades argumentativas) y tipos de interacciones (participantes, tipos de seguimiento de los docentes, posiciones involucradas). 
En esta línea, es posible apreciar que el discurso argumentativo ocurre en la enseñanza de ciencias de una manera muy heterogénea. Esta heterogeneidad es relevante desde el punto de vista de procesos de enseñanza y aprendizaje pues en cada forma de discurso argumentativo ocurren procesos psicológicos distintos. En este sentido, podemos decir que los episodios en que los niños oponen justificadamente sus puntos de vista acerca de procesos científicos llegando a un acuerdo (conocimiento canónico), es posiblemente el escenario ideal desde el punto de vista del aprendizaje de ciencias, pero es sólo uno de las diversas posibilidades en que aparece el discurso argumentativo en el aula.

\section{2. ¿EN QUÉ EXTENSIÓN SE USA EL DISCURSO ARGUMENTATIVO EN LA ENSEÑANZA DE CIENCIAS EN LAS SESIONES OBSERVADAS?}

A partir del cruce de las tres dimensiones antes descritas, se desarrolló una pauta para describir cuantitativamente el tipo de uso de discurso argumentativo en las clases observadas. Como se mencionó anteriormente, se describieron cinco tipos de interacciones que se resumen en la Tabla 2. Cada una de estas interacciones aceptaba tres niveles de uso conceptual (definidos en la Tabla 3) y cinco estructuras argumentativas según presencia de mecanismos semióticos (resumidas en la Tabla 4).

A continuación se reportan los resultados por profesor y sesión.

\begin{tabular}{|c|c|}
\hline Nivel 1 & $\begin{array}{l}\text { Se caracteriza por un uso del lenguaje cotidiano con ausencia de conceptos } \\
\text { científicos. }\end{array}$ \\
\hline Nivel 2 & $\begin{array}{l}\text { Se caracteriza porque los conceptos científicos no se usan relacionados entre } \\
\text { sí desde sus propiedades generales y mecanismos que subyacen la relación. }\end{array}$ \\
\hline Nivel 3 & $\begin{array}{l}\text { Se caracteriza por un uso sistemático de relaciones entre conceptos científicos } \\
\text { según las propiedades de la clase a la que pertenecen. }\end{array}$ \\
\hline
\end{tabular}

Tabla 3. Niveles conceptuales

\begin{tabular}{|c|c|}
\hline $\begin{array}{c}\text { Estructura } \\
\text { argumentativa }\end{array}$ & Definición \\
\hline Justificación & $\begin{array}{r}\text { Cuando episodio de interacción entendido como una unidad temática sólo } \\
\text { incluye demanda de justificación o justificación. }\end{array}$ \\
\hline $\begin{array}{c}\text { Oposición } \\
\text { implícita }\end{array}$ & $\begin{array}{c}\text { Cuando episodio de interacción entendido como una unidad temática incluye } \\
\text { una oposición de ideas que queda implícita en la medida que sólo se trabaja y } \\
\text { reconoce una posición. }\end{array}$ \\
\hline $\begin{array}{c}\text { Oposición } \\
\text { explícita }\end{array}$ & $\begin{array}{c}\text { Cuando episodio de interacción entendido como una unidad temática } \\
\text { incluye una oposición que es reconocida y queda abierta (si es que } \\
\text { hay respuesta ésta no prosigue la elaboración). }\end{array}$ \\
\hline Resolución & $\begin{array}{c}\text { Cuando episodio de interacción entendido como una unidad temática } \\
\text { además de incluir una oposición explícita se resuelve de manera que } \\
\text { los estudiantes muestran acuerdo con la solución. }\end{array}$ \\
\hline
\end{tabular}




\begin{tabular}{|c|c|}
\hline $\begin{array}{c}\text { Resolución } \\
\text { autoritaria }\end{array}$ & $\begin{array}{c}\text { Cuando episodio de interacción entendido como una unidad temática } \\
\text { además de incluir una oposición explícita se resuelve por el profesor } \\
\text { de manera autoritativa sin que los estudiantes muestren grados de } \\
\text { acuerdo o desacuerdo. }\end{array}$ \\
\hline
\end{tabular}

Tabla 4. Tipos de estructuras argumentativas

\subsubsection{Profesor de establecimiento particular}

Las observaciones del discurso de los segmentos de clase completa del único profesor varón se resumen en las Tabla 5, donde se presenta una visión del discurso dedicado al contenido colapsado por tipo de interacción, por nivel conceptual y por nivel argumentativo. Además, se presenta el resumen según segmento interactivo, instrucción directa y trabajo en grupo y el total del discurso dedicado a argumentar. Todo esto, a su vez, considerando duración total, número de ocurrencias, promedio y porcentaje con respecto al total de la clase (en el caso de la argumentación también se presenta como porcentaje del tiempo total dedicado a clase completa dedicada al contenido) para cada una de las observaciones ( 1 y 2$)$.

\begin{tabular}{|c|c|c|c|c|c|c|c|c|}
\hline \multirow[b]{2}{*}{ Observaciones } & \multicolumn{2}{|c|}{ Ocurrencia } & \multicolumn{2}{|c|}{ Duración } & \multicolumn{2}{|c|}{ Promedio } & \multicolumn{2}{|c|}{$\begin{array}{l}\text { Porcentaje del } \\
\text { tiempo total de la } \\
\text { clase }\end{array}$} \\
\hline & 1 & 2 & 1 & 2 & 1 & 2 & 1 & 2 \\
\hline IRE & 1 & - & 0,1 & - & - & - & 0,2 & - \\
\hline IRF & 5 & 18 & 4,4 & 18,1 & 0,9 & 1 & 8,5 & 19,2 \\
\hline IRD & 8 & 9 & 34,4 & 23 & 4,3 & 2,5 & 66 & 24,2 \\
\hline IRI & 1 & 1 & 3,8 & 1,1 & - & - & 7,4 & 1,1 \\
\hline Nivel 1 & - & - & - & - & - & - & - & - \\
\hline Nivel 2 & - & 1 & - & 0,8 & - & - & - & 0,9 \\
\hline Nivel 3 & 18 & 32 & 48 & 49,4 & 1,9 & 1,5 & 92 & 52 \\
\hline Justificativas & 6 & 6 & 14,6 & 10,7 & 2,4 & 1,8 & 28 & 11,3 \\
\hline Oposición implícita & 1 & 4 & 3,8 & 7,1 & - & 1,7 & 7,4 & 7,5 \\
\hline Oposición explícita & - & - & - & - & - & - & - & - \\
\hline Resolución & - & - & - & - & - & - & - & - \\
\hline Resolución autoritaria & 2 & 1 & 17,9 & 1,7 & 9 & - & 34,6 & 1,8 \\
\hline $\begin{array}{l}\text { Total episodios } \\
\text { interacción }\end{array}$ & 15 & 28 & 42,7 & 32,2 & 2,8 & 1,15 & 82,4 & 34,1 \\
\hline $\begin{array}{l}\text { Total instrucción } \\
\text { directa }\end{array}$ & 3 & 5 & 5,1 & 8 & 1,7 & 1,6 & 8,2 & 12,7 \\
\hline $\begin{array}{l}\text { Total trabajo en grupo } \\
\text { y/o individual }\end{array}$ & - & 2 & - & 24,9 & - & 12,4 & - & 39,7 \\
\hline Total argumentación & 8 & 8 & 36,3 & 19,5 & 4,5 & $\overline{4,5}$ & $\begin{array}{l}70 \\
(75,3) *\end{array}$ & $\begin{array}{l}22,6 \\
(48,5)^{*}\end{array}$ \\
\hline
\end{tabular}

*Porcentaje del tiempo clase completa dedicado a contenido.

Tabla 5. Resumen por dimensiones y observaciones profesor PS/7º básico (Fuerzas) 
Se aprecia que en la primera sesión el profesor trató los contenidos en su mayoría de manera interactiva $(82,4 \%)$, cuya totalidad fue trabajada a un nivel conceptual taxonómico (nivel 3). Además, la mayoría de los episodios interactivos fueron episodios en los que participó más de un estudiante (IRD+IRI), cuya duración promedio fue de 4 minutos. Gran parte de la conversación involucró algún nivel de discurso argumentativo, el que en total representó un 75,3\% del total de la clase dedicada a contenido. Incluso, casi la mitad del total de discurso argumentativo (36,3 minutos) se dedicó a episodios en los que, además de justificación (la que apareció ampliamente), hubo oposición explícita y algún tipo de resolución, siendo estos episodios bastante extensos según se puede apreciar en la Tabla 5. Ahora bien, el discurso argumentativo predominante se caracterizó por interacciones en los que participaba más de un estudiante, pero cuyo discurso se dirigía y dialogaba con el del profesor y no con otros estudiantes. Además, si bien los episodios resolutivos predominaron en extensión, la resolución en ambos casos fue una resolución unidireccional del profesor. En este sentido, estos episodios se caracterizaron por el intercambio del profesor y los estudiantes, cuya oposición de ideas y perspectivas más que discutida fue sancionada por el profesor.

Este patrón se mantiene en la sesión dos dentro de los segmentos de clase completa dedicados a trabajar contenido. Sólo considerando los segmentos de clase completa dedicados a trabajar contenido, también el $80 \%$ se dedicó a episodios interactivos prácticamente en un $100 \%$ a nivel conceptual taxonómico, según se aprecia en la Tabla 5. Sin embargo, a diferencia de la sesión anterior en la mayoría de los episodios interactivos participó un estudiante (IRF). Ahora, aunque los episodios en los que participaron más de un estudiante ocurrieron menos, se extendieron por más tiempo en total ( $23 \mathrm{v} / \mathrm{s} \mathrm{18,2} \mathrm{minutos).} \mathrm{Del} \mathrm{total} \mathrm{de}$ los segmentos de clase completa dedicados a contenido un $48,5 \%$ involucraron discurso argumentativo. Por último, el discurso argumentativo predominante fue el justificativo y en segundo lugar la oposición implícita, ocurriendo sólo un episodio breve con resolución autoritaria.

Lo que se puede concluir de la comparación entre ambas sesiones es que el profesor muestra una clara tendencia a usar los conceptos en la clase de una manera conceptual taxonómica, predominando la interacción entre el profesor y los estudiantes. Es decir, más que una discusión, la interacción toma una forma tradicional de diálogo pedagógico entre los estudiantes y el profesor donde la asimetría juega un rol esencial. Esto se evidencia en la baja ocurrencia de episodios de oposición explícita y ausencia total de resolución no autoritaria. Esto quiere decir que si se presentó oposición explícita entre ideas y puntos de vista, estos fueron en mayor medida entre el profesor y los estudiantes y la resolución no fue producto del diálogo. De esta manera, si bien la argumentación apareció no en pocas ocasiones en las sesiones observadas del profesor, ésta se caracterizó por una baja exploración de visiones contradictorias y opuestas, estando al servicio de un desarrollo conceptual orientado hacia la apropiación de una única y verdadera posición científica.

\subsubsection{Profesora de establecimiento particular}

Las sesiones observadas de la profesora que se desempeñaba en el nivel de 5to básico en el mismo establecimiento particular subvencionado, se resumen en las Tabla 6.

En la primera sesión la profesora trató los contenidos en gran parte de manera interactiva, pero representando un porcentaje mucho menor del total de la clase que las sesiones del profesor antes descrito (37,2\% v/s 82,4\%). Ahora bien, destaca un trabajo 
de los contenidos a nivel conceptual taxonómico pero, a diferencia del profesor antes caracterizado, también dedica un porcentaje considerable de la sesión a trabajar contenidos en un nivel conceptual no taxonómico (nivel 2). Con respecto a los episodios interactivos, dedica más tiempo a interacciones en las que participa más de un estudiante (13,5 minutos), pero igualmente destacan las interacciones en las que participa un solo estudiante (10,7 minutos en total). No se aprecian interacciones en las que los estudiantes discuten sus ideas entre sí (IRI). Por último, se aprecia una extensión mucho menor de uso de discurso argumentativo, pues sólo un 22,4\% del tiempo dedicado a trabajar contenido en clase completa involucra algún tipo de argumentación. En general, ésta no aparece en la instrucción directa sino sólo en los episodios interactivos y sólo en un nivel justificativo.

\begin{tabular}{|c|c|c|c|c|c|c|c|c|}
\hline \multirow[b]{2}{*}{ Observaciones } & \multicolumn{2}{|c|}{ Ocurrencia } & \multicolumn{2}{|c|}{ Duración } & \multicolumn{2}{|c|}{ Promedio } & \multicolumn{2}{|c|}{$\begin{array}{l}\text { Porcentaje del tiempo } \\
\text { total de la clase }\end{array}$} \\
\hline & 1 & 2 & 1 & 2 & 1 & 2 & 1 & 2 \\
\hline IRE & 5 & 1 & 2,2 & 2,3 & 0,4 & - & 2,1 & 3,7 \\
\hline$\overline{\mathrm{IRF}}$ & 7 & 17 & 8,5 & 13,9 & 1,2 & 0,8 & 13,1 & 22,2 \\
\hline IRD & 8 & 4 & 13,5 & 8,5 & 1,7 & 2,1 & 20,8 & 13,6 \\
\hline IRI & - & - & - & - & - & - & - & - \\
\hline Nivel 1 & - & - & - & - & - & - & - & - \\
\hline Nivel 2 & 9 & 3 & 13,6 & 4,5 & 0,7 & 1,5 & 19 & 7,2 \\
\hline Nivel 3 & 19 & 28 & 23,4 & 32,4 & 1,2 & 1,15 & 34,6 & 52 \\
\hline Justificativas & 4 & 1 & 8,1 & 1,9 & 2 & - & 12,4 & 3,1 \\
\hline Oposición implícita & - & - & - & - & - & - & - & - \\
\hline Oposición explícita & - & - & - & - & - & - & - & - \\
\hline Resolución & - & - & - & - & - & - & - & - \\
\hline Resolución autoritaria & - & 1 & - & 3,6 & - & - & - & 5,8 \\
\hline $\begin{array}{l}\text { Total episodios } \\
\text { interacción }\end{array}$ & 20 & 22 & 24,2 & 24,4 & 1,2 & 1,1 & 37,2 & 38,9 \\
\hline $\begin{array}{l}\text { Total instrucción } \\
\text { directa }\end{array}$ & 7 & 9 & 11,9 & 12,2 & 1,7 & 1,35 & 18,3 & 19,4 \\
\hline $\begin{array}{l}\text { Total trabajo en grupo } \\
\text { y/o individual } \\
\end{array}$ & 1 & 7 & 4,7 & 12,5 & - & 1,8 & 7,7 & 20 \\
\hline Total argumentación & 4 & 2 & 8,1 & 5,5 & 2 & 2,7 & $\begin{array}{l}12,4 \\
(22,4)^{*}\end{array}$ & $\begin{array}{l}8,9 \\
(15)^{*}\end{array}$ \\
\hline
\end{tabular}

Tabla 6. Resumen por dimensiones y observaciones profesora PS/5º básico (Reinos seres vivos)

La segunda sesión de la misma profesora sigue un patrón similar (ver Tabla 6). Los episodios predominantes de interacción son IRF e IRD, tendiendo a ser los primeros más cortos en duración. En esta ocasión, trabaja más marcadamente el contenido a un nivel conceptual taxonómico. El discurso argumentativo es muy discreto (15\%) e involucra, además de justificativas, un episodio relativamente largo de resoluciones autoritarias.

En esta línea, el estilo discursivo de las sesiones observadas se caracteriza por interacciones relativamente cortas en las que participa un solo estudiante, a un nivel mayormente conceptual taxonómico, con inexistente formulación de oposición tanto a nivel implícito como explícito. Además, mantiene interacciones jerárquicas con pocas ocasiones para que los estudiantes elaboren opiniones (por la corta duración de las interacciones) y se refieran a las opiniones de otros estudiantes (por la inexistencia de IRI). 


\subsubsection{Profesora de establecimiento municipal}

Las sesiones observadas de la profesora que se desempeñaba en el establecimiento municipal de la zona norponiente de Santiago en el nivel de 5to básico, se resumen en la Tabla 7.

Podemos apreciar que en la primera sesión la profesora trató los contenidos con la ausencia de episodios de instrucción directa. Además, la mayoría del tiempo las interacciones involucraron a más de un estudiante y se aprecia una extensión considerable (en comparación con las observaciones de los otros profesores) dedicada a interacciones en los que el estudiante se dirigía a otro estudiante. Sin embargo, a diferencia también de los profesores antes analizados, esta profesora trabaja el contenido prioritariamente a nivel conceptual no taxonómico y en segunda medida no conceptual. Sólo un 5\% de los segmentos de clase completa dedicada al contenido usa conceptos a nivel taxonómico. Por último, se aprecia una extensión amplia del uso de discurso argumentativo $(62,6 \%$ segmentos clase completa dedicada a contenido). Sin embargo, a diferencia de los profesores antes analizados, la estructura argumentativa predominante es el de oposición implícita. Destaca la aparición de un episodio de oposición explícita y uno de resolución no autoritaria.

\begin{tabular}{|c|c|c|c|c|c|c|c|c|}
\hline \multirow[b]{2}{*}{ Observaciones } & \multicolumn{2}{|c|}{ Ocurrencia } & \multicolumn{2}{|c|}{ Duración } & \multicolumn{2}{|c|}{ Promedio } & \multicolumn{2}{|c|}{$\begin{array}{l}\text { Porcentaje del tiempo } \\
\text { total de la clase }\end{array}$} \\
\hline & 1 & 2 & 1 & 2 & 1 & 2 & 1 & 2 \\
\hline IRE & 8 & 1 & 0,8 & 0,1 & 0,5 & - & 0,9 & 0,2 \\
\hline IRF & 3 & - & 3,1 & - & 0,9 & - & 6,9 & - \\
\hline IRD & 14 & 7 & 25 & 15,1 & 1,4 & 2,2 & 32 & 23 \\
\hline IRI & 4 & 6 & 4,4 & 12,4 & 2 & 2,1 & 13 & 19 \\
\hline Nivel 1 & 5 & 13 & 3,1 & 24,8 & 1,7 & 1,9 & 14 & 37 \\
\hline Nivel 2 & 20 & 1 & 28 & 2,2 & 1,1 & - & 35 & 3,1 \\
\hline Nivel 3 & 2 & 1 & 2,1 & 2,7 & 1,3 & - & 3,7 & 4,1 \\
\hline Justificativas & 3 & 2 & 6,4 & 1,9 & 1,7 & 0,9 & 8,4 & 2,8 \\
\hline Oposición implícita & 7 & 3 & 1 & 9,3 & 1,7 & 3,1 & 19 & 14 \\
\hline Oposición explícita & 1 & 1 & 4,4 & 0,9 & - & - & 3,5 & 1,4 \\
\hline Resolución & 1 & 3 & 1 & 8 & - & 2,7 & 4,2 & 12 \\
\hline Resolución autoritaria & - & 1 & - & 2,2 & - & - & - & 3,3 \\
\hline $\begin{array}{l}\text { Total episodios } \\
\text { interacción }\end{array}$ & 29 & 14 & 33,3 & 27,6 & 1,2 & $\mathbf{0 , 9}$ & 56 & 22,2 \\
\hline $\begin{array}{l}\text { Total instrucción } \\
\text { directa }\end{array}$ & - & 1 & - & 2,2 & - & - & - & 3,1 \\
\hline $\begin{array}{l}\text { Total trabajo en grupo } \\
\text { y/o individual }\end{array}$ & 2 & 3 & 22,5 & 23,4 & 11,8 & 7,6 & 38,2 & 35,7 \\
\hline Total argumentación & 12 & 10 & 12,8 & 15,1 & 1,8 & 1,5 & $\begin{array}{l}35 \\
(62,6) *\end{array}$ & $\begin{array}{l}23 \\
(50,6) *\end{array}$ \\
\hline
\end{tabular}

Tabla 7. Resumen por dimensiones y observaciones profesora $\mathrm{M} / 5^{\circ}$ básico (Estímulos y sensaciones)

El patrón discursivo anterior se repite en la segunda observación de la profesora en este nivel, tal como observamos en la Tabla 7. La profesora trató los contenidos en los segmentos de clase completa en más de un $90 \%$ del tiempo de manera interactiva, predominando las interacciones en las que participaban más estudiantes, muchas veces 
dirigiéndose a opiniones de otros estudiantes (IRI) predominantemente a nivel no conceptual. Esto sugiere un estilo discursivo con amplia presencia de discusión (simetría) e indagación, pero a muy bajo nivel conceptual. Probablemente a esto se debe el bajo nivel de interacciones justificativas y de oposición explícita, predominando episodios en los que se pregunta por acuerdo o desacuerdo pero sobre cuestiones en las que no caben dos perspectivas.

Las observaciones de la misma profesora en séptimo básico se resumen en la Tabla 8. En la primera sesión, la profesora trató nuevamente los contenidos sin episodios de instrucción directa. Además, la mayoría del tiempo las interacciones involucraron a más de un estudiante quienes dirigían sus contribuciones a la profesora. Sólo un 6,3\% de los segmentos de clase completa dedicada al contenido usa conceptos a nivel taxonómico. Por último, se aprecia una menor de uso de discurso argumentativo (38,4\% segmentos clase completa dedicada a contenido), predominando esta vez el nivel de justificación.

\begin{tabular}{|c|c|c|c|c|c|c|c|c|}
\hline \multirow[b]{2}{*}{ Observaciones } & \multicolumn{2}{|c|}{ Ocurrencia } & \multicolumn{2}{|c|}{ Duración } & \multicolumn{2}{|c|}{ Promedio } & \multicolumn{2}{|c|}{$\begin{array}{l}\text { Porcentaje del tiempo } \\
\text { total de la clase }\end{array}$} \\
\hline & 1 & 2 & 1 & 2 & 1 & 2 & 1 & 2 \\
\hline IRE & 2 & - & 4,3 & - & 0,4 & - & 1 & - \\
\hline IRF & 6 & 4 & 2,6 & 2,3 & 0,5 & 0,6 & 3,9 & 3,6 \\
\hline IRD & 12 & 15 & 20 & 19 & 2 & 1,3 & 31,6 & 19 \\
\hline IRI & 2 & 2 & 7,9 & 6,4 & 2,2 & 3,2 & 5,6 & 10,2 \\
\hline Nivel 1 & 3 & 1 & 8,7 & 0,5 & 1,4 & - & 3,9 & 0,9 \\
\hline Nivel 2 & 11 & 9 & 21,7 & 12 & 0,9 & 1,3 & 35,4 & 19,2 \\
\hline Nivel 3 & 1 & 13 & 2,6 & 21,5 & - & 1,6 & 2,7 & 31,6 \\
\hline Justificativas & 3 & 1 & 5,2 & 0,3 & 2,1 & - & 8 & 0,4 \\
\hline Oposición implícita & 1 & - & 11,8 & - & - & - & 1,3 & - \\
\hline Oposición explícita & 2 & - & 2,2 & - & 2,2 & - & 5,5 & - \\
\hline Resolución & 1 & 2 & 2,6 & 6,4 & - & 3,2 & 1,3 & 10,2 \\
\hline Resolución autoritaria & - & - & - & - & - & - & - & - \\
\hline $\begin{array}{l}\text { Total episodios } \\
\text { interacción }\end{array}$ & 22 & 21 & 34,8 & 27,7 & 1,5 & 1,3 & 42 & 44 \\
\hline $\begin{array}{l}\text { Total instrucción } \\
\text { directa }\end{array}$ & - & 2 & - & 8,3 & - & 4,1 & - & 10,8 \\
\hline $\begin{array}{l}\text { Total trabajo en grupo } \\
\text { y/o individual }\end{array}$ & 4 & 4 & 23,6 & 6,2 & 5,5 & 1,5 & 25,9 & 9,8 \\
\hline Total argumentación & 7 & 3 & 21,8 & 6,7 & 1 & 1,15 & $\begin{array}{l}16,2 \\
(38,4)^{*}\end{array}$ & $\begin{array}{l}10,6 \\
(18,6)^{*}\end{array}$ \\
\hline
\end{tabular}

Tabla 8. Resumen por dimensiones y observaciones profesora $\mathrm{M} / 7^{\circ}$ básico (Sistema Reproductivo)

La segunda observación en este nivel muestras ciertas variaciones. Principalmente, destaca el uso no menor de instrucción directa que esta vez ocupa un $23 \%$ de los segmentos de clase completa dedicadas al contenido. Además, hay un uso extenso de nivel conceptual taxonómico el que representa casi un $60 \%$ de este mismo segmento. La interacción sigue estando ampliamente presente en un $77 \%$ del tiempo predominando aquella en la que participa más de un estudiante. Por último, el discurso argumentativo está mucho menos presente notándose un aumento del nivel de resolución no autoritaria. 
Esta diferencia sugiere dos aproximaciones diferentes. En la primera observación, la profesora trabajó el contenido básicamente en torno a la discusión en clase completa (asimétrica) y en el trabajo en grupos, con un bajo nivel conceptual, pero un amplio uso de discurso argumentativo. Se trató de indagar y discutir experiencias y descripciones sin demanda de justificación, abriendo la posibilidad del desacuerdo. En la segunda observación, en este mismo nivel la profesora fue más directiva y taxonómica y, si bien hubo discusión, cada episodio fue más breve.

Las observaciones en ambos niveles muestran ciertas similitudes. Una de ellas fue el amplio uso de la interacción y, aunque el discurso argumentativo no predomina, sí aparecen episodios que no aparecen en otros profesores en la misma extensión como la resolución no autoritaria y oposición implícita. En tres de las cuatro observaciones el nivel conceptual usado se mueve entre sentido común y conceptual no taxonómico. Llama la atención que en la observación en que hay un uso conceptual más alto, la discusión y argumentación baja considerablemente.

Sin embargo, entre los niveles igualmente se marca un uso conceptual diferente. Mientras en 5 to básico hay una importante tendencia al sentido común-no taxonómico, en $7^{\circ}$ se tiende hacia el uso conceptual. Así mismo, mientras en 5to se observan muchos más episodios en los que los estudiantes reaccionan a la perspectiva de otros estudiantes, en $7^{\circ}$ estos prácticamente desaparecen instalándose una relación en la interacción más asimétrica.

\section{CONCLUSIONES}

Los datos confirman aquello que la tradición en investigación en argumentación en la enseñanza de ciencias sugiere (Erduran y Jimenez-Aleixandre, 2008): que la argumentación en aula es una cuestión compleja. El cruce de las dimensiones identificadas da cuenta de una diversidad inmensa de procesos de argumentación en el aula. Si bien las observaciones informan que la argumentación tal y como es entendida por van Eemeren y Grootendorst (1992), es decir, como una actividad discursiva en la que hay puntos de vista opuestos que intentan alcanzar un acuerdo, es escasa, éste no es el caso si es que se es sensible a la diversidad del discurso argumentativo en el aula. El porcentaje de tiempo en que se verificaron procesos de argumentación con resolución y que incluía discusión de ideas contradictorias (ya sea estudiantes o estudiantes y profesor) es relativamente escaso. Sin embargo, el uso de discurso argumentativo ampliamente entendido apareció en mucha mayor medida.

Las dimensiones identificadas y sus cruces evidencian la diversidad de unidades de discurso argumentativo que pueden encontrarse en el aula de ciencias. Esto es un desafío para estudios a mayor escala, ya que cualquier intento de aprehender la diversidad y complejidad del uso de discurso argumentativo para la enseñanza de ciencias debería lidiar con esta diversidad de 'unidades' y eventualmente 'códigos'.

Más aún, estudios a mayor escala debieran considerar que estas distinciones son particularmente relevantes desde el punto de vista del aprendizaje en ciencias, pues los procesos psicológicos que ocurren en el discurso argumentativo, según ocurran en diferentes tipos de unidades, son diferentes. Por ejemplo, considerando el nivel conceptual, no es equivalente en términos de aprendizaje una interacción en la que se discuten 
cuestiones de la vida cotidiana (siendo de todas maneras importantes) con una en la que se discuten explicaciones científicas, siendo estas últimas centrales para el aprendizaje de ciencias. Autores como Lemke (1990) y Mortimer y Scott (2003), por ejemplo, describen el aprendizaje de ciencias como la posibilidad de los estudiantes de hablar de las ciencias, y esto se juega en gran medida en la posibilidad de trascender el sentido común e incorporar conceptos científicos en su discurso.

Por otro lado, los procesos psicológicos también varían según las estructuras de argumentación que se ponen en juego. Si bien se entiende que la mera demanda de justificación desafía al pensamiento del estudiante a buscar elementos de apoyo para una idea (desafía a pensar más que meramente a recordar de manera mecánica), es la presencia de la oposición explícita la que gatilla procesos de pensamiento reflexivo en el movimiento de defenderse de esa oposición (en los que el pensamiento tiene como objeto el mismo pensamiento) que desembocan en aprendizaje (ver estudios de Howe, 2010). En la misma línea, una respuesta que reconoce una oposición explícita y provee de alguna salida permite que el estudiante no sólo se quede pensando en la oposición sino que entienda cómo ésta se resuelve desde el punto vista del conocimiento canónico de las ciencias (cuya apropiación es el fin de la enseñanza escolar).

Por último, con respecto al tipo de interacciones teóricamente se tendería a pensar, siguiendo los trabajos de Piaget (1926/1962) y Bárbara Rogoff (1994), que las interacciones en las que hay estructuras de argumentación que incluyen la oposición y se dan entre participantes con una relación simétrica (estudiantes), promoverían en mayor grado el aprendizaje en la medida que permitirían una apropiación genuina por parte del estudiante del conocimiento canónico. Sin embargo, y como veremos a continuación, este tipo de interacciones, aunque se observan, no son frecuentes en todos los docentes. Por otro lado, dado que el fin de argumentar en el aula es apropiarse de un conocimiento que no está abierto, sino ya construido (Leitao, 2008), la guía experta por parte del profesor parece una cuestión central. Tal y como platea Howe (2009), es muy difícil que estudiantes solos reconstruyan el conocimiento escolar por mucho que argumenten complejamente. De esta manera, los procesos argumentativos asimétricos, es decir, entre estudiantes y profesores, no sólo es más frecuente, sino relevante desde el punto de vista del aprendizaje.

En base a este cruce, y dado los resultados cuantitativos, podría ser posible en estudios a larga escala rastrear la tendencia de un mismo profesor, de distintos profesores o distintos niveles etarios, a ubicarse en una distribución tridimensional, según los ejes mencionados (estructura argumentativa, interaccional y nivel conceptual). Los análisis cuantitativos sugieren que podrían haber diferencias por niveles en el trabajo conceptual predominante: en séptimo básico se mostró una tendencia hacia un uso conceptual taxonómico. Es interesante reparar en cómo la profesora del establecimiento municipal en los momentos en que usaba los conceptos a nivel alto o taxonómico, disminuía la riqueza de la estructura argumentativa de las interacciones discursivas, y al contrario cómo éstas propendían a discusiones simétricas cuando el nivel de uso conceptual disminuía. A la vez, es interesante notar cómo las interacciones argumentativas también se enriquecían en estructura cuando las interacciones eran simétricas (interacción entre estudiantes).

Sería interesante que futuros estudios indagaran acerca de una tendencia predominante del uso argumentativo según profesor y nivel. Más aún, a partir de la identificación de estas tendencias, se podrían entregar elementos para interpretar las condiciones que influyen en las habilidades científicas que desarrollan los estudiantes chilenos. Si la tendencia 
predominante es un uso de argumentación en interacciones asimétricas y cerradas, de alto nivel conceptual, entonces se entendería que los estudiantes desarrollen un manejo más bien mecánico de los conceptos científicos. Por el contrario, si la tendencia predominante fuera a un uso de argumentación en interacciones abiertas en las que los estudiantes discuten puntos de vista opuestos entre ellos, pero con un bajo nivel conceptual, se podría entender que éstos desarrollasen habilidades de pensamiento y argumentación, pero no pensamiento científico. Por último, y aún cuando éste tipo de tendencia no se observó, si la actividad discursiva ocurriera predominantemente en interacciones abiertas, con estructuras argumentativas complejas y de un alto nivel conceptual, se esperaría que los estudiantes desarrollasen habilidades de pensamiento científico avanzado.

Cabe destacar que el presente estudio no se centra en la calidad de argumentación de los estudiantes, como la mayoría de los estudios de argumentación en el aula, sino que contribuye en la comprensión de las interacciones argumentativas orales tal como se desarrollan espontáneamente, y en las oportunidades que se les da a los estudiantes para argumentar en el aula. En conclusión, este estudio aporta en la identificación de dimensiones con sus respectivos niveles, que son relevantes a la hora de estudiar a larga escala la ocurrencia de procesos de argumentación oral en la enseñanza de ciencias, y de entender la relación entre procesos de argumentación y aprendizaje de las ciencias.

\section{REFERENCIAS BIBLIOGRÁFICAS}

Alexander, R.J. (2001). Culture and Pedagogy: international comparisons in primary education. Oxford and Boston: Blackwell.

Asterhan, C. S. C., \& Schwarz, B. B. (2009). Argumentation and explanation in conceptual change: Indications from protocol analyses of peer-to-peer dialog. Cognitive Science, vol. 33, n.3, 374-400.

Asterhan, C. S. C., \& Schwarz, B. B. (2007). The effects of monological and dialogical argumentation on concept learning in evolutionary theory. Journal Of Educational Psychology, vol. 99, n.3, 626-639.

Bajtín, M. (2002). El Problema de los géneros discursivos. En M, Bajtín, Estética de la creación verbal (248-293). Buenos Aires: Siglo XXI editores.

Billig, M. (1987). Arguing and Thinking: A rhetorical approach to social psychology. Cambridge: Cambridge University Press.

Burton, R. \& Flammang, C. (2001), Amélioration de l'enseignement des sciences au premier degré secondaire. Pour une approche constructiviste de l'apprentissage. Informations pédagogiques, vol. 52, n.2, 29-40.

Candela, A. (1999). Ciencia en el aula. Los alumnos entre la argumentación y el consenso. México: Paidós.

Cazden, C. B. (2001). Classroom discourse: The language of teaching and learning. Portsmouth, NH: Heinemann.

De Chiaro, S. \& Leitão, S. (2005). O papel do profesor na construcão discursiva da argumentacão em sala de aula [Teacher's role in a discursive construction of classroom argumentation]. Psicologia: Reflexão e Crítica, vol. 18, n.3 ,350-357.

Driver, R., Newton, P., \& Osborne, J. (2000). Establishing the norms of scientific argumentation in classrooms. Science Education, vol. 84, n.3, 287-312.

Duschl, R. \& Osborne, J. (2002) Supporting and Promoting Argumentation Discourse in Science Education. Studies in Science Education, vol.38, n.1, 39-72. 
Eemeren, F.H. Van, \& Grootendorst, R. (1992). Argumentation, communication, and fallacies: A pragma-dialectical perspective. Hillsdale, NJ: Lawrence Erlbaum Associates.

Erduran, S. (2008). Methodological Foundations in the Study of Argumentation in Science Classrooms. In S. Erduran \& M. P. Jimenez-Aleixandre, Argumentation in science education: Perspectives from classroom-based research (pp. 47-70). Netherlands: Springer.

Forman, E. A. (2000). Knowledge building in discourse communities. Human development, vol. 43, n.6, 364-368.

Galton, M., Hargreaves, L., Comber, C., Pell, T, \& Wall, D. (1999). 'Inside the Primary Classroom: 20 Years On', London: Routledge.

Hammer, D., Russ, R., Mikeska, J., \& Scherr, R. (2008). Identifying inquiry and conceptualizing students' abilities. In R. Duschl \& R. Grandy, Establishing a Consensus Agenda for K-12 Science Inquiry (pp. 138-156). Rotterdam, NL: Sense Publishers.

Howe, C. (2010). Peer dialogue and cognitive development. A two way relationship?. In K. Littleton Y C. Howe, Educational dialogues: Understanding and promoting productive interaction (pp. 32-47). London: Routledge.

Howe, C. (2009). Expert support for group work in elementary science: the role of consensus. In B. Schwarz, T. Dreyfus \& R. Hershkowitz, Transformation of knowledge through classroom Interaction (pp. 93-104). New York: Routledge

Howe, C., Tolmie, A., Thurston, A., Topping, K., Christie, D., Livingston, K., Jessiman, E. \& Donaldson, C. (2007). Group work in elementary science: towards organisational principles for supporting pupil learning. Learning and Instruction, vol.17, n. 5, 549-563.

Jimenez-Aleixandre, M. P., Rodrigues, A. B., \& Duschl, R. (2000). "Doing the lesson" or "doing science": Argument in high school genetics. Science Education, vol.84, n.6, 757-792.

Jimenez-Aleixandre, P., \& Erudran, S. (2008). Argumentation in science education: An overview. In S. Erduran Y M. P. Jimenez-Aleixandre, Argumentation in science education: Perspectives from classroom-based research (pp. 47-70). Netherlands: Springer.

Kelly, G. J., \& Takao, A. (2002). Epistemic levels in argument: An analysis of university oceanography students' use of evidence in writing. Science Education, vol.86, n.3, 314-342.

Kelly, G. J., \& Chen, C. (1999). The sound of music: Constructing science as a sociocultural practices through oral and written discourse. Journal of Research in Science Teaching, vol.36, n.8, 883-915.

Kelly, G. J., Druker, S., \& Chen, C. (1998). Students' reasoning about electricity: Combining performance assessments with argumentation analysis. International Journal of Science Education, vol.20, n.7, 849-871.

Kim, H., \& Song, J. (2006). The features of peer argumentation in middle school students' scientific inquiry. Research in Science Education, vol.36, n.3, 211-233.

Kuhn, D., \& Udell, W. (2003). The development of argument skills. Child Development, vol.74, n.5, $1245-1260$.

Larrain, A. (2009). El rol de la argumentación en la alfabetización científica. Estudios Públicos, vol.116, n.4, 167-193.

Leitão, S. (2000). The Potential of Argument of knowledge Building. Human Development, vol.43, n.6, 332-360.

Leitão, S. (2002). Self-argumentation: The rhetorical dimension of meta-cognition. Trabajo presentado en "Kitchen Seminar" de Francis Hiatt School of Psychology, Clark University, MA. Novembro.

Leitão, S. (2008). Arguing and Learning. In C. Lightfood Y M. Lyra, Challenges and strategies for studying human development in cultural contexts (pp. 221-251). Rome: Firera publishing.

Lemke, J., L. (1990). Talking science: Language, learning and values. Westport: Ablex Publishing.

Mercer, N. (2009). Developing argumentation: Lessons learned in the primary school. In N. Muller Mirza Y A.N. Perret-Clermont, Argumentation and Education (pp. 177-194). New York: Springer. 
Mercer, N., Dawes, R., Wegerif, R., \& Sams, C. (2004) Reasoning as a scientist: ways of helping children to use language to learn science. British Educational Research Journal, vol.30, n.3, 367-385.

Mineduc (2010). Resumen de resultados Pisa 2009 Chile. Simce, Unidad de currículum y evaluación. Extraído de www.simce.cl el 4 de marzo de 2011.

Mortimer, E. \& Scott, P. (2003) Meaning making in secondary science classrooms. Maidenhead and Philadelphia: Open University Press.

Norris, S.P. \& Phillips, L.M. (2002). How literacy in its fundamental sense is central to scientific literacy. Science Education, vol.87, n.2, 224-240.

Nussbaum, E. M., Y Sinatra, G. M. (2003). Argument and conceptual engagement. Contemporary Educational Psychology, vol.28, n.3. 384-395.

Nystrand, M., A. Gamoran, R. Kachur, C. Prendergast. (1997). Opening dialogue: Understanding the dynamics of language and learning in the English classroom. New York: Teachers College Press.

Orange, C. (2003). Débat scientifique dans la classe, problématisation et argumentation: le cas d'un débat sur la nutrition au cours moyen. Aster, vol.37, 83-107.

Osborne, J. F., Erduran, S., \& SIMON, S. (2004). Enhancing the Quality of Argument in School Science. Journal of Research in Science Teaching, vol.41, n.10, 994-1020.

Perelman, Ch., \& Olbrechts-Tyteca, L. (1989). Tratado de la Argumentación. La Nueva Retórica. Madrid: Gredos.

Piaget, J. (1962). The Language and Thought of the Child (M. Gabain, Trans.). Cleveland, OH: Meridian. (Original work published 1926).

Preiss, D. (2009). The Chilean instructional pattern for the teaching of language: a video-survey study based on a national program for the assessment of teaching. Learning and Individual Differences, vol.19, n.1, 1-11.

Rojas-Drummond, S., \& Peon Zapata, M. (2004). Exploratory talk, argumentation and reasoning in mexican primary school children. Language and Education, vol.18, n.6, 539-557.

Preiss, D. (2010). Folk Pedagogy and Cultural Markers in Teaching: Three Illustrations from Chile. In Preiss, D. Y Sternberg, R.J. Innovations in educational psychology: Perspectives on Learning, Teaching, and Human Development (pp. 325-356). New York: Springer Publishing Company.

Roth, K., Druker, S., Garnier, H., Lemmens, M., Chen, C., Kawanaka, T., Rasmussen, D., Trubacova, S., \& Warvi, D. (2006). Teaching science in five countries. Results from the TIMSS 1999 video study. Washington, DC: U.S. Government Printing Office.

Sandoval,W. A. \& Millwood, K. (2005). The quality of students' use of evidence in written scientific explanations. Cognition and Instruction, vol.23, n.1, 23-55.

Schwarz, B. (2009). Argumentation and learning. In N. Muller Mirza Y A.N. Perret-Clermont, Argumentation and Education (pp. 91-126). New York: Springer.

Stigler, J. W., Gonzales, P., Kawanaka, T., Knoll, S., Y Serrano, A. (1999). The TIMSS Videotape Classroom Study: methods and findings from an exploratory research project on eighth-grade mathematics instruction in Germany, Japan, and the United States (Research and Development Report No. NCES 1999-074). Washington, D.C.: U.S. Government Printing Office.

Toulmin, S. (1958). The uses of argument. Cambridge: Cambridge University Press.

Wertsch, I. V. (1998). La mente en acción. Buenos Aires: Aique.

Yore, L. D., Bisanz, G. L., \& Hand, B. M. (2003). Examining the literacy component of science literacy: 25 years of language arts and science research. International Journal of Science Education, vol.25, n.6, 689-725.

Zohar, A. \& Nemet, F. (2002). Fostering students knowledge and argumentation skills through dilemmas in human genetics. Journal of Research in Science Teaching, vol.39, n.1, 35-62. 